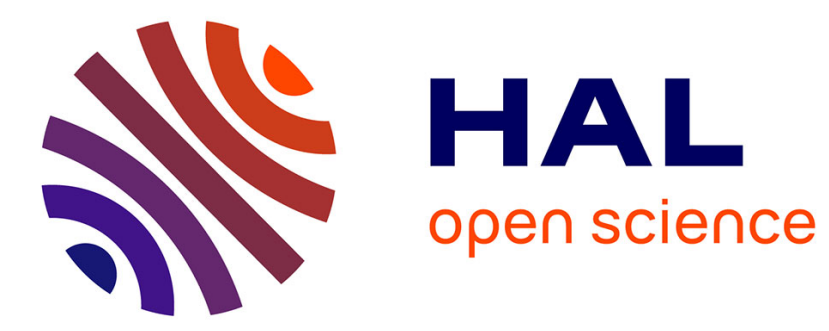

\title{
Scènes de composition oratoire
}

\author{
Jean-Michel David
}

\section{To cite this version:}

Jean-Michel David. Scènes de composition oratoire. Revue des études anciennes, 1990, 92, pp.259-264. hal-01090071

\section{HAL Id: hal-01090071 https://hal.science/hal-01090071}

Submitted on 8 Dec 2014

HAL is a multi-disciplinary open access archive for the deposit and dissemination of scientific research documents, whether they are published or not. The documents may come from teaching and research institutions in France or abroad, or from public or private research centers.
L'archive ouverte pluridisciplinaire HAL, est destinée au dépôt et à la diffusion de documents scientifiques de niveau recherche, publiés ou non, émanant des établissements d'enseignement et de recherche français ou étrangers, des laboratoires publics ou privés. 


\title{
SCÈNES DE COMPOSITION ORATOIRE
}

\author{
J.-M. DAVID*
}

Résumé. - Le rapprochement entre quelques descriptions littéraires (en particulier Cic., De or., III, 17) et des représentations figurées permet de reconstituer les conditions concrètes qui entouraient le travail de composition oratoire, d'y retrouver la trace de l'art de la mémoire et d'apprécier la valeur de cette mise en spectacle de la réflexion intellectuelle.

Abstract. - The comparison of some textual descriptions (e. g. Cic., De or., III, 17) with some pictures let us reconstitute the actual conditions of the rhetoric construction, find traces of the art of memory and valute this exhibition of the thought.

De tous les livres du De oratore, le troisième était sans doute le plus important. Il permettait à Cicéron de faire exposer par L. Licinius Crassus les principales définitions qui lui tenaient à coeur, de l'éloquence et de ses rapports avec la philosophie. Aussi prenait-il soin de bien marquer par la mise en scène elle-même toute l'importance de ce qui allait y être dit. Le soin notamment, avec lequel Crassus avait préparé son discours, était très précisément souligné.

«Cotta (...) fut surtout frappé de ce que Crassus, durant tout ce temps de la sieste était resté plongé dans une réflexion très tendue et très profonde. Connaissant bien l'expression de sa physionomie, lorsqu'il devait plaider, et la fixité de son regard, lorsqu'il réfléchissait, signes quil avait souvent observés en lui à la veille de procès importants, Cotta avait profité du moment où les autres reposaient pour venir dans l'exèdre où Crassus était étendu sur le lit qu'on lui avait dressé ; l'ayant trouvé tout absorbé par ses réflexions, il s'était retiré aussitôt. Près de deux heures s'écoulèrent dans le même silence'. "

L'intérêt de ce passage ne se limite pas à la mise en scène du De oratore. Il donne en effet une image saisissante de l'orateur au milieu de son travail de composition, puisque Cicéron soulignait que l'état dans lequel Cotta avait trouvé Crassus était aussi celui dans lequel il l'avait vu préparer ses plus grands plaidoyers.

* Université des Sciences humaines de Strasbourg.

1. Cic., De or., III, 17 : (...) In primis hoc a se Cotta animadversum esse dicebat omne illud tempus meridianum Crassum in acerrima atque attentissima cogitatione posuisse seseque, qui voltum eius, cum ei dicendum esset, optutumque oculorum in cogitando probe nosset atque in maximis causis saepe vidisset, tum dedita opera quiescentibus aliis in eam exedram venisse, in qua Crassus posito lectulo recubuisset, cumque eum defixum esse in cogitatione sensisset, statim recessisse atque in eo silentio duas horas fere esse consumptas (trad. E. Courbaud). 
Plusieurs traits méritent alors d'être relevés, parce qu'en les rapprochant d'autres documents, on peut évaluer ce qu'étaient les habitudes des Romains en matière de travail intellectuel et saisir surtout l'importance de la représentation de l'orateur à l'ouvrage dans la définition de son statut.

La disposition du corps tout d'abord : la position allongée qui aurait rendu certainement très inconfortable un effort d'écriture prolongé, « la réflexion très tendue et très profonde » (acerrima atque attentissima cogitatio), « la fixité du regard lorsqu'il réfléchissait » (obtutus oculorum in cogitando) n'étaient pas des attitudes aussi naturelles qu'il pourrait y sembler à première lecture. D'autant qu'à suivre la description de Cicéron, Crassus demeura ainsi pendant plus de deux heures, complètement absorbé par ses réflexions et les menant sans bouger jusqu'au terme de l'élaboration de son exposé.

L'explication tient sans doute à ce que les méthodes de travail des Anciens étaient complètement différentes des nôtres. Il leur était impossible de composer leurs discours par écrit. Les tablettes ne permettaient pas de noter autre chose que des textes un peu brefs. Les volumina étaient trop malcommodes pour pouvoir être facilement utilisés. Seule la mémoire permettait de retenir un développement d'une certaine durée. Et l'on peut gager que l'effort auquel se livrait Crassus était celui qui consistait à composer sa conférence et à en fixer dans son esprit le plan, les idées et les figures afin de pouvoir les restituer sans peine lorsque le moment viendrait de la tenir devant ses amis.

On connaît assez bien, grâce aux manuels de rhétorique, la technique qui permettait d'y parvenir $^{2}$. Il s'agissait de se représenter mentalement un lieu précis et bien connu (une basilique ou une maison, par exemple), d'y définir des points précis (les colonnes d'un portique ou les angles d'un atrium), de composer et de disposer là des images qui rappelleraient les arguments ou les expressions que l'on souhaitait utiliser, par les attributs dont on les avait pourvues (une coupe pour un empoisonnement par exemple, ou des tablettes pour un testament) et enfin de mémoriser l'ensemble ${ }^{3}$. Au moment de prononcer le discours, on reparcourait en pensée l'itinéraire selon lequel on les avait placées et en reprenant tous les éléments, on retrouvait les idées, les métaphores et les formules que l'on avait préparées. Cela demandait un gros effort de concentration qui allait bien au-delà de celui de la stricte composition. Quintilien le soulignait : « l'attention joue un rôle très important en cette matière, et elle est, si j'ose dire, comme l'acuité du regard qui ne se détourne pas des objets qu'elle considère ${ }^{4}$. " Si bien que l'on doit imaginer que cette représentation de Crassus tendu et absorbé par son travail était en même temps un signe de la puissance et de la qualité de sa réflexion.

Les indications que Cicéron donnait sur le cadre matériel de la scène allaient dans le même sens. Crassus se tenait dans une exèdre, allongé sur un lit. 1969.

2. Cf. F. YATES, L'art de la mémoire, Paris, 1975, et surtout H. BLUM, Die antike Mnemotechnik, Hildesheim,

3. Cf. Rh. Her., III, 28-40 ; Cic., De or., II, 350-360 ; Part. or., 26 ; Quint., XI, 2.

4. Quint. XI, 2, $10:$ Nec dubium est quin plurimum in hac parte valeat mentis intentio et velut acies luminum a prospectu rerum, quas intuetur, non aversa (trad. J. Cousin). 
Le lieu avait son importance. Il s'agissait d'une petite pièce s'ouvrant probablement sur un portique, dans la partie la plus privée de la maison ${ }^{5}$. C'est là que l'on se retirait pour s'isoler ou pour entretenir une conversation avec des amis choisis ${ }^{6}$. Vitruve citait en effet les exèdres avec les conclavia et les triclinia ${ }^{7}$ et soulignait que ces pièces étaient de celles où il n'était permis d'entrer que sur invitation ${ }^{8}$. En ce sens, elles ne se distinguaient pas de la plupart des autres cubicula pour lesquels on employait un esclave, un cubicularius, dont la fonction était précisément d'écarter les importuns et de faire respecter la volonté du maître de rester seul'9.

Les pièces de la maison n'avaient pas de fonction prédéfinie. Parfois, il arrivait que l'on associât un cubiculum à une bibliothèque ${ }^{10}$. Mais ce n'était pas la règle. Ces espaces retirés pouvaient tout aussi bien servir de lieu de repos que de travail. Ils étaient simplement les lieux de l'isolement et du silence nécessaire à la concentration ou au sommeil ${ }^{11}$.

Le meuble, lectulus, répondait à une définition du même ordre. Il s'agissait d'un petit lit qui pouvait servir pour le repos, mais dont l'emploi pour le travail intellectuel est bien attesté. Outre ce passage du De oratore, on pourrait citer quelques allusions de poètes au lieu où ils avaient coutume de composer leurs vers ${ }^{12}$, ainsi qu'une indication de Suétone qui, à propos d'Auguste, distinguait entre le lit où l'empereur travaillait et celui où il dormait ${ }^{13}$. Mais on retiendra surtout deux extraits des lettres de Pline décrivant des amis disparus qui avaient coutume de se tenir ainsi : «On lui apportait la salutation, on l'entourait ; le plus souvent sur son lit de travail, dans une chambre toujours remplie de visiteurs que n'attirait pas sa fortune, il passait son temps en savants entretiens quand il n'était pas occupé à écrire $»^{14}$.

La façon dont Pline rappelait l'image du personnage dont il faisait ainsi l'éloge funèbre n'est certainement pas innocente. Elle contribuait à valoriser un souvenir en le grandissant par l'évocation d'une activité intellectuelle.

Ce fragment permet ainsi un autre rapprochement. Les scènes de ce genre ne sont pas rares sur les monuments funéraires. H. I. Marrou en avait étudié certaines autrefois et en avait bien noté le caractère réaliste ${ }^{15}$. Les objets, les volumina en particulier, jouaient le rôle d'attributs de culture qui qualifiaient le défunt comme un homme supérieur, héroïsé en quelque sorte par la fréquentation des Muses. Certes la plupart de ces représentations étaient assez tardives, mais il ne semble pas que cela ôte de la pertinence à la comparaison avec les descriptions de Cicéron et de Pline, car les comportements qu'elles évoquaient étaient de ceux qui s'inscrivent dans la longue durée.

5. Cf. pour une définition et des exemples P. GrIMAL, Les jardins romains, $3^{\text {e }}$ ed., Paris, 1984, p. 215 ; 269 ; et de façon plus précise B. TAMM, Auditorium and Palatium, Lund, 1963, p. 128-147; S. SETTIS, « Esedra » e « ninfeo » nella terminologia architettonica del mondo romano, $A N R W, \mathrm{I}, 4,1973$, p. 673-675.

6. Cf. aussi De Nat. Deor., I, 15.

7. VI, 3,8 .

8. VI, 5,1 .

9. Cf. Cic., 2 Verr., III, 8 ; Att., VI, $2,5$.

10. Pline, Ep., II, 17, 7 ; cf. Vitr., I, 2,7 ; VI, $4,1$.

11. Varr., L. L., IX, 58 ; Sen., Ep., 72, 2.

12. Catulle, 50, 14-16 ; Hor., Sat., I, 4, 133-134 ; Ovide, Ars Amat., III, 541-542 ; Trist., I, 11, $37-38$.

13. Suét., Div. Aug., 78, 2.

14. Pline, Ep., III, 7, 4 : salutabatur, colebatur multumque in lectulo iacens, cubiculo semper non ex fortuna frequenti, doctissimis sermonibus dies transigebat, cum a scribendo vacaret; V, 5, 5 cf. aussi Val. Max., VIII, 7, 3.

15. Mousikos Anêr, Etudes sur les scènes de la vie intellectuelle figurant sur les monuments funéraires romains, Grenoble, 1937. 


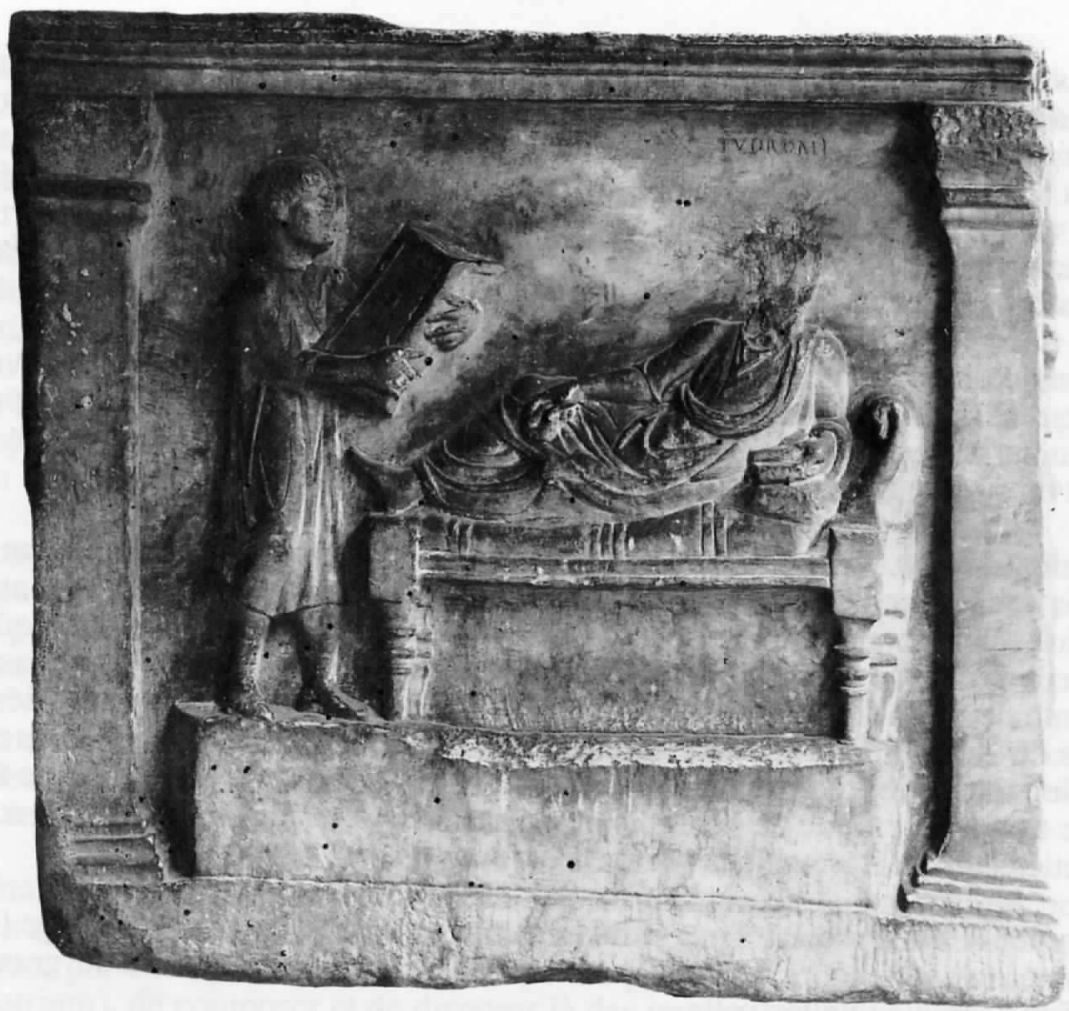

Fig. 1. - Sarcophage de C. Valerius Petronianus, Musée archéologique de Milan.

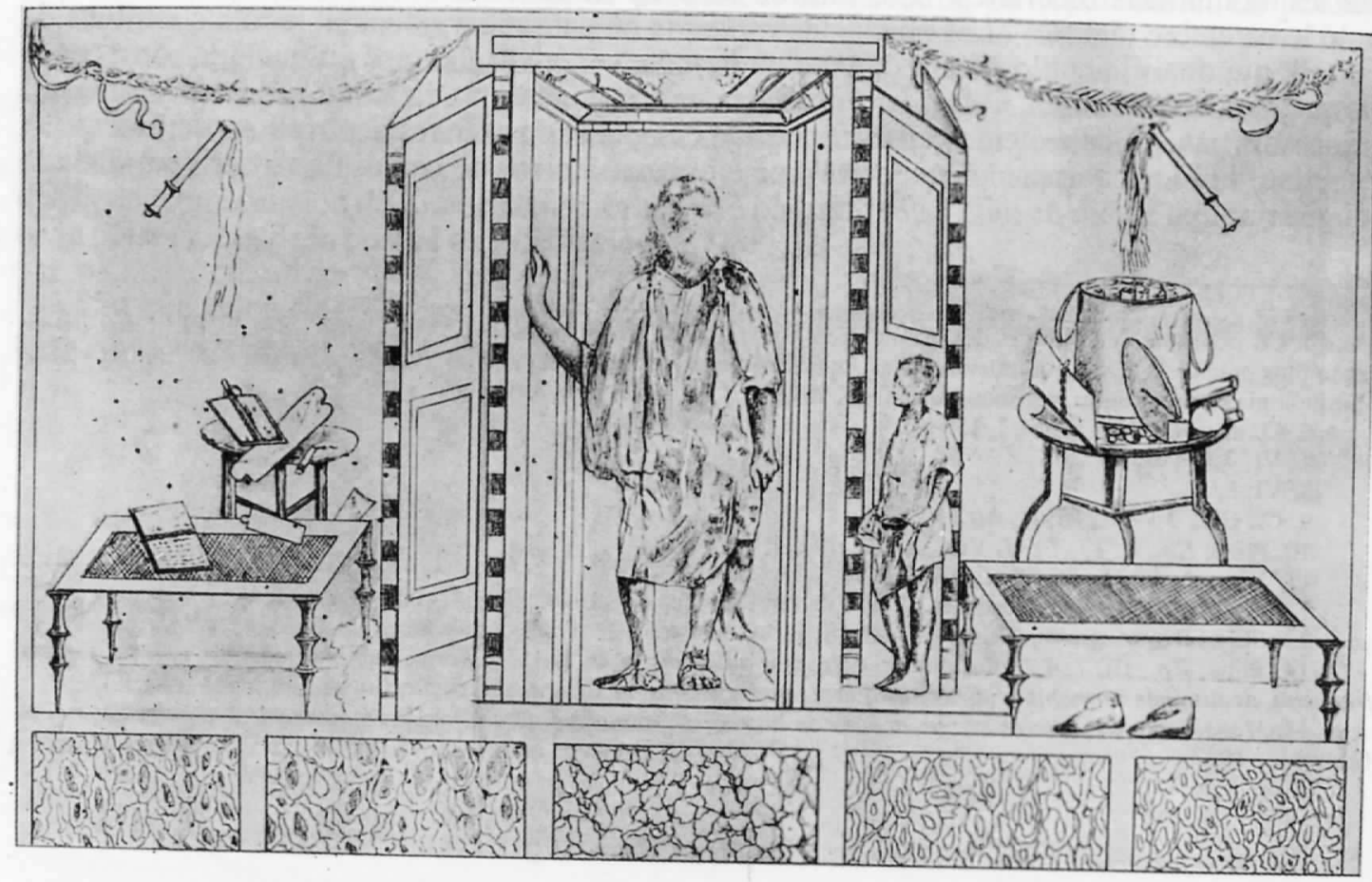

Fig. 2. - D'après G. Spano. La tomba dell'edile C. Vestorio Prisco in Pompei, MAL, VII, 3, 1943, p. 237-315, fig. 9, p. 273. 
Un monument, en tout cas, doit retenir l'attention. Il s'agit du sarcophage de C. Valerius Petronianus de Milan, qui date au plus tôt du troisième siècle de notre ère ${ }^{16}$. L'inscription qui y était portée définissait ce jeune homme, mort à vingt-trois ans, comme un causidicus et soulignait qu'il était déjà allé cinq fois à Rome ou ailleurs pour y plaider la cause de sa cité ${ }^{17}$. Il était représenté plaidant devant un magistrat, un préteur sans doute, sur le côté droit du sarcophage. Sur le côté gauche, il apparaissait en toge, allongé sur un lit tenant un volumen de la main gauche et sans doute une mappa de la main droite, pendant que sur la gauche un homme en tunique s'avançait vers lui en portant une sorte de caisse ou de boîte à archives.

L'opposition entre les deux scènes en donnait la signification. Le même homme qui prononçait un discours sur celle de droite, le préparait sur celle de gauche ${ }^{18}$. L'homme en tunique était un esclave qui apportait les dossiers qui lui étaient nécessaires. Lui-même continuait de manifester par ses attributs, la toge et le volumen notamment, sa fonction d'orateur. Le lit et la position allongée rappelaient enfin que tout ceci se déroulait à l'intérieur de la maison à l'écart, dans l'espace privé d'un cubiculum. La scène en fin de compte indiquait, au même titre que celle du plaidoyer et que l'inscription qui énumérait les titres du défunt, que celui-ci était un avocat important, compétent et capable qui avait su bien défendre les intérêts de ses concitoyens.

Une représentation analogue confirme d'ailleurs le mécanisme sémantique de l'opposition entre les deux aspects, public et privé, d'une même fonction. Il s'agit du tombeau bien connu de l'édile de Pompéi, C. Vestorius Priscus, antérieur de peu à l'éruption du Vésuve ${ }^{19}$. Sur la face principale du monument, une peinture le montrait siégeant sur son tribunal, entouré d'appariteurs et de plaideurs. Or, sur la face strictement opposée, on le voyait, accueilli par un petit esclave (un cubicularius, peut-être), pénétrer à l'intérieur de la maison, dans une pièce meublée de deux lits et de tabourets recouverts d'objets divers : tablettes, volumina et capsa $a^{20}$. L'accumulation des instruments du travail intellectuel confortait ici l'image principale du magistrat en fonction : elle signifiait sa diligentia, son ardeur à l'ouvrage et particulièrement à l'étude des textes qui lui permettait d'être à la hauteur de ses responsabilités juridictionnelles. Ces deux représentations confirment et illustrent bien ce que les textes de Cicéron et de Pline nous avaient déjà permis de savoir. Et pas seulement du point de vue au fond assez anecdotique des méthodes et des habitudes romaines en matière de réflexion intellectuelle. Elles incitent en effet à considérer que certaines indications étaient plus précises et plus riches de sens qu'on ne le

16. Fig. 1. Cf. H. Gabelmann, Die Werkstattgruppen der oberitalischen Sarkophage, Bonn, 1973, $\mathrm{n}^{\circ} 111$, qui donne la bibliographie antérieure, et du même auteur, Antike Audienz- und Tribunalszenen, Darmstadt, 1984, $\mathrm{n}^{\circ}$ 93, p. 194-195. Cf. aussi dans ces deux mêmes publications le sarcophage retenu sous les $\mathrm{n}^{\circ} 61$ et 92 respectivement qui aurait bien pu être composé de la même façon mais dont la partie opposée à la scène de tribunal a disparu.

17. CIL, V, 5894 : D(is) m(anibus) C. Valeri Petroniani / decur(ionis), pontif(icis), sacerd(otis) / iuven(um). Med(iolaniensium) causidic(i) / quinq(uies) gratuit(o) legation(ibus) urbic(is) et peregrin(is) pro re p(ublica) sua funct(i). I Vixit ann(is) XXIII, mens(ibus) VIIII, d(iebus) XIIII. I C. Valerius Eutychianus / VIvir aug(ustalis), pater I fil(io) incompar(abili) et sibi.

18. L'interprétation d'H. GabelmanN, Tribunalszenen, p. 195 qui y voyait l'opposition entre l'otium et le negotium me paraît moins pertinente.

19. Fig. 2. Cf. G. Spano, La tomba dell'edile C. Vestorio Prisco in Pompei, MAL, VII, 3, 1943, p. $289-294$; p. 246 date le monument des demières décennies de Pompei ; cf. depuis, J. M. Dentzer, La tombe de C. Vestorius Priscus dans la tradition de la peinture italique, MEFR, LXXIV, 1962, p. 533-594; B. M. FELLETTI MAJ, La tradizione italica nell'arte romana, Rome, 1977, p. 327-329; P. Castrén, Ordo Populusque Pompeianus, Rome, 1975, p. 120 (75-76 p. C.) ; H. GABELMANN, Tribunalszenen, $\mathrm{n}^{\circ}$ 95, p. 198-199 et J. RonKE, Magistratische Repräsentation im Römischen Relief, Londres, 1987, p. 141-142; 681.

20. À la différence de ce que pensait G. SPANO, o. c., p. 277 et J. M. DENTZER, o. c., p. 574, je pense que la maison ainsi représentée est la maison réelle et non le séjour des morts. 
pense souvent ${ }^{21}$. Elles ne renvoyaient pas de façon uniforme et indéfinie à la culture ou à l'intelligence, mais évoquaient avec précision des pratiques intellectuelles et les donnaient en spectacle. Elles contribuaient ainsi à établir fermement la supériorité de ceux qui s'y livraient.

Le savoir et la compétence n'étaient pas donnés à tous. Surtout quand il s'agissait de ceux qui permettaient de jouer le rôle de protecteur et de défenseur de ses concitoyens. Les montrer, les manifester, même au détour d'un éloge ou d'une description, revenait en fait à revendiquer pour ceux qui les possédaient, un statut élevé dans la cité.

21. On pourrait peut-être ainsi enrichir la lecture d'un sarcophage du troisième siècle que citait déjà H. I. MARROU, o. $c ., \mathrm{n}^{\circ} 18$ et 134, p. 50 et 124 , et qui a été étudié plus récemment par M. WEGNER, Die Musensarkophage, Berlin, 1966, $n^{\circ} 139$, p. 58. Il représente le même enfant sous deux aspects différents. Sur la frise du sarcophage, le jeune défunt est assis, nu sous le pallium, il tient dans la main gauche un volumen et lève la main droite; sur le couvercle, ses gestes sont les mêmes, mais il est allongé, en toge, et tourne la tête vers le lointain. M. Wegner notait déjà que la tenue qui lui était attribuée sur la frise, faisait probablement référence à la philosophie grecque. Ne faudrait-il pas voir alors dans celle qui était la sienne sur le couvercle, et qui correspond si bien aux attitudes que l'on a déjà décrites, une référence à la thétorique qui complèterait la première dans une définition plus générale de la paideia? 